Received: 2015.01.12 Accepted: 2015.02.02 Published: 2015.05 .04
Authors' Contribution: Study Design A Data Collection B Statistical Analysis C Data Interpretation D Manuscript Preparation E Literature Search F Funds Collection $G$
Corresponding Author: Source of support:

\section{Analysis of Bile Colonization and Intestinal Flora may Improve Management in Liver Transplant Recipients Undergoing ERCP}

\author{
ABCDEF 1 lyad Kabar* \\ ABCDEF 1 Anna Hüsing* \\ ABDE 1 Vito R. Cicinnati \\ ABCDF 1 Laura Heitschmidt \\ BCEF 1 Susanne Beckebaum \\ BCD 2 Gerold Thölking \\ ABEF 1 Hartmut H. Schmidt \\ ACD 3 Helge Karch \\ ABDEF 3 Frank Kipp
}

* These authors contributed equally to this work and are co-first authors lyad Kabar, e-mail: iyad.kabar@ukmuenster.de Departmental sources

Background: Immunosuppression, denervation of biliary tract, and presence of biliary strictures favor colonization of bile with microorganisms after liver transplantation. Little is known about spectrum and antibiotic susceptibility of this colonization.

Material/Methods: Bile and feces were collected prospectively from 38 patients who underwent endoscopic retrograde cholangiopancreaticography after liver transplantation. Samples were analyzed for colonization and antibiotic susceptibility.

Results: $\quad$ From the 38 tested bile samples, $86.6 \%$ tested positive. Of those, 26 (78.8\%) were polymicrobial. Of isolated bile samples, 52 (64.2\%) were gram-positive, $22.2 \%$ were gram-negative, and $13.6 \%$ revealed Candida albicans. Most detectable gram-positive bacteria were Enterococcus faecium. Most detectable gram-negative bacteria were E. coli and Klebsiella pneumonia. Our analyses revealed high resistance rates of the isolates. Only $55.6 \%$ of isolates were sensitive to ciprofloxacin, $54 \%$ were sensitive to piperacillin/tazobactam, and $60.3 \%$ were sensitive to imipenem. High susceptibility rates were found for linezolid and vancomycin $(72.9 \%$ and $72.6 \%$, respectively). We found a high correlation between microorganisms found in bile and those isolated from stool.

Conclusions: Bile of liver transplant recipients is frequently colonized with microorganisms. The starting point of this colonization is usually the intestine. Systematic analysis of bile colonization during endoscopic interventions on biliary tracts of liver transplant recipients might help to select effective prophylactic antibiotic regimes as well as to facilitate the choice of suitable antimicrobial therapy in case of septic complications.

MeSH Keywords: Bile Duct Diseases • Cholangiopancreatography, Endoscopic Retrograde • Liver Transplantation

Full-text PDF: http://www.annalsoftransplantation.com/abstract/index/idArt/893549

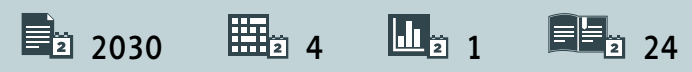




\section{Background}

Biliary complications after liver transplantation (LT) are common and remain a major source of morbidity; thus, they significantly influence patient and organ survival [1]. In recent years, endoscopic retrograde cholangiopancreatography (ERCP) has superseded surgery as the gold standard in management of bile duct complications after LT [2]. However, ERCP still is an invasive tool and may have serious complications such as pancreatitis, perforation, bleeding, and septic complications $[2,3]$.

Infectious complications are among the most common causes of death after ERCP $[4,5]$. Septic complications after ERCP include bacteremia, sepsis, ascending cholangitis, and liver abscess. A potential focus for infectious complications may be bactibilia.

Although bile is sterile in healthy individuals [6,7], bactibilia is frequently found in patients after LT. Additional risk factors for colonization of bile include the presence of foreign bodies such as stones and stents $[8,9]$.

Immunosuppression and occurrence of biliary strictures after LT favor colonization of bile with microorganisms. Little is known about the clinical relevance of this colonization, but preliminary data show that colonization of bile with bacteria and fungi is associated with development of septic complications after endoscopic interventions on the bile duct [10]. However, recent antibiotic prophylaxis of ERCP-induced septic complications is based on recommendations in the non-transplant setting. Furthermore, there are currently no guidelines for the treatment of septic complications after endoscopic biliary interventions in liver transplant recipients. This is especially important because most of these patients have had multiple antibiotic therapies in the course of their liver disease.

Therefore, the aim of this study was to evaluate microbiological spectrum and antibiotic resistance of specimens found in bile of liver-transplanted individuals who underwent ERCP, in order to investigate the clinical efficacy of antibiotics administered to prevent infectious complications. Since there is evidence that enteric flora represents a reservoir for ascending cholangitis and further infectious diseases, particularly after LT [11,12], we additionally analyzed enteric flora and the correlation between isolates found in bile and stool cultures in our patient population.

\section{Material and Methods}

This prospective study was carried out between May 2011 and September 2013 at the University Hospital of Münster. Thirtyeight patients (25 (65.8\%) were male and 13 (34.2\%) were female, mean age was $52.2 \pm 11.4$ years) were enrolled into the study after LT. Table 1 shows patients' characteristics and
Table 1. Demographic and clinical characteristics and endoscopic findings in the $38 \mathrm{LT}$ recipients.

\begin{tabular}{|c|c|c|}
\hline N & \multicolumn{2}{|r|}{38} \\
\hline Male & 25 & $(65.8 \%)$ \\
\hline Female & 13 & $(34.2 \%)$ \\
\hline Age, yr, mean $\pm S D$ & \multicolumn{2}{|c|}{$52.2 \pm 11.4$} \\
\hline \multicolumn{3}{|l|}{ Etiology of liver disease } \\
\hline Alcohol & \multicolumn{2}{|r|}{15} \\
\hline $\mathrm{HCC}$ & \multicolumn{2}{|r|}{8} \\
\hline Hepatitis $B / C$ virus & \multicolumn{2}{|r|}{5} \\
\hline Cholestatic liver diseases & \multicolumn{2}{|r|}{5} \\
\hline Other & \multicolumn{2}{|r|}{5} \\
\hline $\begin{array}{l}\text { Time from transplant to } \\
\text { ERCP, months }\end{array}$ & \multicolumn{2}{|c|}{25.6 (range $0.3-164.2$ ) } \\
\hline \multicolumn{3}{|l|}{ Endoscopic findings } \\
\hline AS & 13 & $(44.8 \%)$ \\
\hline NAS & 4 & $(13.8 \%)$ \\
\hline Biliary leakage & 4 & $(13.8 \%)$ \\
\hline Stones & 1 & (3.4\%) \\
\hline ITBL & 1 & $(3.4 \%)$ \\
\hline Biliary cast & 1 & $(3.4 \%)$ \\
\hline Non & 5 & $(17.2 \%)$ \\
\hline
\end{tabular}

HCC - hepatocellular carcinoma; AS - anastomotic stricture; NAS - non-anastomotic stricture, ITBL - ischemic type biliary lesion.

endoscopic findings. All patients gave written informed consent and treatment was performed in adherence to the guidelines of the Declaration of Helsinki. The trial was approved by the local ethics committee. Indications for ERCP were increasing liver and cholestatic parameters with or without an intraor extra-hepatic biliary dilatation seen on imaging. All patients were treated with at least 1 immunosuppressant. Twentyseven $(71.1 \%)$ were on calcineurin-inhibitor-based immunosuppression (57.9\% on tacrolimus and $13.1 \%$ on ciclosporin), and eight $(21.1 \%)$ were on $\mathrm{m}$-TOR-based immunosuppression (13.2\% everolimus and $7.9 \%$ sirolimus). Two patients (5.3\%) were maintained on tacrolimus in combination with everolimus and 1 patient (2.6\%) received mycophenolate mofetil only.

Bile was collected either via percutaneous biliary drainage (PTC) in nine patients and during ERCP in 29 patients. Endoscopic retrograde cholangiopancreaticography was performed using a therapeutic duodenoscope (TJF-180V, Olympus Corp., Tokyo, Japan). The bile duct was accessed with wire-guided cannulation 
using a sterile sphincterotome (0.035 inch, THSF-35-480, Cook Medical INC.; Tri-Tome, TRI 20, Cook Medical, Inc.). Bile was aspirated immediately after cannulation of the bile duct before contrast material was administered. Approximately $2-5 \mathrm{~mL}$ of bile were collected and immediately submitted to microbiological laboratory analysis in a sterile syringe. Stool samples were also collected from each patient and submitted to the microbiological laboratory in the same way.

Bile was streaked out on Columbia blood agar, McConkey agar, Kimmig agar, and boiled blood agar under increased $\mathrm{CO}_{2}$ atmosphere (5-10\%) to detect aerobic growth. To detect anaerobic microorganisms, we used Schaedler agar and ThioglycolateBouillon. All samples were incubated at $35^{\circ}$ to $37^{\circ} \mathrm{C}$. Plates were examined after $24 \mathrm{~h}$ and $48 \mathrm{~h}$. For identification, we used matrix-assisted laser desorption ionization time-of-flight mass spectrometry (MALDI-TOF MS, microflex ${ }^{\odot}$, Bruker Daltonik $\mathrm{GmbH}$, Bremen). Susceptibility testing was performed by Vitek 2 (bioMerieux ${ }^{\odot}$, Nürtingen, Germany).

If bile specimens showed any growth of microorganisms, stool specimens were investigated in the same way. Only microorganisms previously found in bile cultures were identified and tested for antibiotic susceptibility.

\section{Antibiotic prophylaxis}

Antibiotics were routinely administered prophylactically in all patients who received ERCP; 18 out of 29 patients $(62.1 \%)$ received ciprofloxacin $(2 \times 750 \mathrm{mg}$ daily), 2 patients $(6.9 \%)$ received imipenem $(3 \times 500 \mathrm{mg}$ daily), and nine patients $(31.0 \%)$ received piperacillin ( $3 \times 4 \mathrm{~g}$ daily) administered in combination with tazobactam $(3 \times 0.5 \mathrm{mg}$ daily). Administration of antimicrobial prophylaxis was started 2 hours before ERCP and was continued for 3 days after intervention. The selection of antibiotics for prophylactic use was made by the responsible attending physician.

\section{Statistical analysis}

Statistical analysis was performed using SPSS Statistics 20 for Windows. Data are presented as mean \pm standard deviation and percentages for categorical variables. Comparison of data was performed using Fisher's exact test. A 2-sided $p<0.05$ was considered significant.

\section{Results}

Endoscopic findings of the 29 patients who received ERCP were anastomotic strictures in 13 cases (44.8\%), non-anastomotic strictures in 4 (13.8\%), biliary leakage in 4 (13.8\%), stones in $1(3.4 \%)$, biliary cast in $1(3.4 \%)$, and normal biliary tree in 5 patients $(17.2 \%)$.

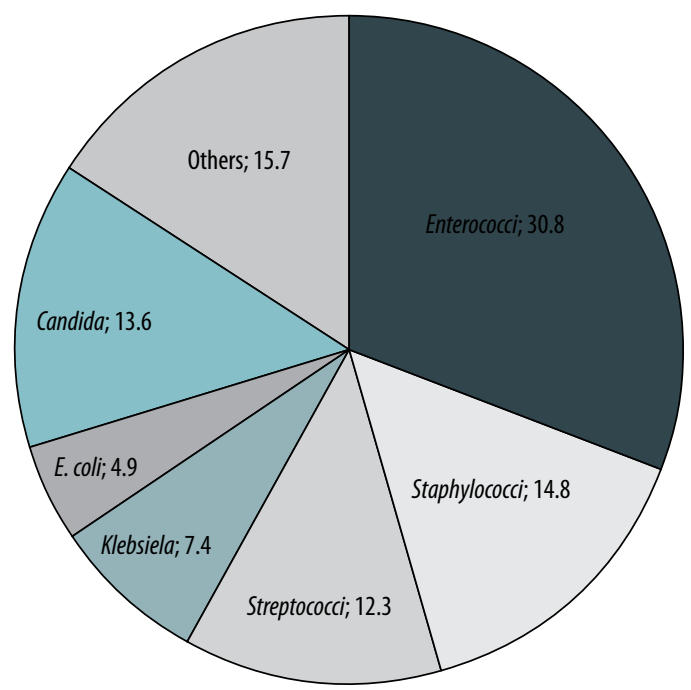

Figure 1. Groups of microorganisms found in bile [\%].

From the 38 bile samples obtained, 33 (86.8\%) bile cultures tested positive and 5 (13.2\%) showed no microbial growth. Seven $(21.2 \%)$ of the positive bile cultures showed only 1 isolate and $26(78.8 \%)$ were polymicrobial. A total of 81 microorganisms were isolated; 52 (64.2\%) gram-positive, 18 (22.2\%) gram-negative, and 11 (13.6\%) Candida albicans. Most common gram-positive isolates were Enterococcus faecium (13.6\%), Enterococcus faecalis (12.3\%), Staphylococcus haemolyticus (7.4\%), and Staphylococcus epidermidis (7.4\%). Most common gram-negative isolates were Escherichia coli (4.9\%) and Klebsiella pneumoniae (4.9\%). There was no difference concerning the microbial spectrum and their resistogram between the isolates from bile collected during ERCP and during PTC. Groups of microorganisms found in bile are shown in Figure 1.

Three patients developed fever in the time between 2 days before and 10 days after ERCP. In all 3, isolates from blood were the same as those found in bile.

According to our results, the only risk factor for bile colonization was a previous endoscopic sphincterotomy, which that was pre-existent in 21 patients $(55.3 \%)(p=0.012)$. Table 2 shows isolates from bile in patients with and without pre-existing endoscopic sphincterotomy. We found no statistical correlation between endoscopic findings and/or stent placement and the colonization of bile ( $p>0.05)$.

\section{Antibiotic susceptibility in bile}

Bacterial isolates were tested for antibiotics typically used for prophylaxis of septic complications after ERCP (ciprofloxacin, imipenem, and piperacillin/tazobactam) and also for vancomycin and linezolid. Susceptibility to ciprofloxacin was found 
Table 2. Microorganisms isolated from bile in patients with and without pre-existing EST.

\begin{tabular}{|c|c|c|c|}
\hline Isolates & Total & Patients with EST & Patients without EST \\
\hline Enterococcus faecium & 11 & 8 & 3 \\
\hline Enterococcus faecalis & 10 & 9 & 1 \\
\hline Enterococcus avium & 4 & 3 & 1 \\
\hline Staphylococcus haemolyticus & 6 & 4 & 2 \\
\hline Staphylococcus epidermidis & 6 & 2 & 4 \\
\hline Escherichia coli & 4 & 4 & 0 \\
\hline Klebsiella pneumoniae & 4 & 3 & 1 \\
\hline Klebsiella oxytoca & 2 & 2 & 0 \\
\hline Streptococcus parasanguinis & 2 & 0 & 2 \\
\hline Streptococcus salivarius & 2 & 0 & 2 \\
\hline Streptococcus anginosus & 2 & 2 & 0 \\
\hline Streptococcus sanguinis & 1 & 1 & 0 \\
\hline Streptococcus oralis & 1 & 0 & 1 \\
\hline Streptococcus pneumoniae & 1 & 0 & 1 \\
\hline Streptococcus viridans & 1 & 0 & 1 \\
\hline Stenotrophomonas maltophilia & 2 & 0 & 2 \\
\hline Hafnia alvei & 1 & 1 & 0 \\
\hline Bacillus spp. & 1 & 0 & 1 \\
\hline Lactobacillus gasseri & 1 & 1 & 0 \\
\hline Lactobacillus rhamnosus & 1 & 0 & 1 \\
\hline Enterobacter cloacae & 1 & 1 & 0 \\
\hline Pseudomonas aeruginosa & 1 & 0 & 1 \\
\hline Pseudomonas stutzeri & 1 & 0 & 1 \\
\hline Corynebacterium jeikeium & 1 & 1 & 0 \\
\hline Micrococcus luteus & 1 & 0 & 1 \\
\hline Kocuria palustris & 1 & 0 & 1 \\
\hline Proteus mirabilis & 1 & 1 & 0 \\
\hline Candida & 11 & 10 & 1 \\
\hline Total & 81 & 53 & 28 \\
\hline
\end{tabular}

EST - endoscopic sphincterotomy.

in $55.6 \%$ of cases, to piperacillin/tazobactam in $54.0 \%$, to imipenem in $60.3 \%$, to vancomycin in $72.6 \%$, and to linezolid in $72.9 \%$. All bacteria with imipenem resistance were gramnegative. One vancomycin-resistant enterococcus (VRE) and 1 bacterium with extended-spectrum beta-lactamase (ESBL) were isolated.

Only $70.8 \%$ of isolates collected during ERCP were sensitive to given antibiotic prophylaxis. Had the patients received either linezolid or vancomycin additionally, an additional $25 \%$ of bacteria $(95.8 \%$ in total) would have been covered by the antibiotic therapy.

Of the tested bacteria, $33.3 \%$ were resistant to all 3 antibiotics used (ciprofloxacin, imipenem, and piperacillin/combactam) and $41.2 \%$ were sensitive to all 3 antibiotics. An add-on administration of linezolid or vancomycin would have covered a total of $90.5 \%$ of all resistant bacteria. 
Table 3. Antibiotic resistance of microorganisms.

\begin{tabular}{|c|c|c|c|c|c|c|}
\hline \multirow{2}{*}{ Antibiotics } & \multicolumn{2}{|c|}{ Sensitive isolates } & \multicolumn{2}{|c|}{ Intermediate isolates } & \multicolumn{2}{|c|}{ Resistant isolates } \\
\hline & Number & $\%$ & Number & $\%$ & Number & $\%$ \\
\hline Ciprofloxacin & 35 & 55.6 & 1 & 1.6 & 27 & 42.9 \\
\hline Piperacillin/tazobactam & 34 & 54.0 & 4 & 6.3 & 25 & 39.7 \\
\hline Imipenem & 38 & 60.3 & 1 & 1.6 & 24 & 38.1 \\
\hline Sulfamethoxazole/trimethoprim & 15 & 23.8 & 1 & 1.6 & 47 & 74.6 \\
\hline Cefotaxime & 22 & 34.9 & 1 & 1.6 & 40 & 63.5 \\
\hline Erythromycin & 13 & 21.0 & 4 & 6.5 & 45 & 72.6 \\
\hline Linezolid & 43 & 72.9 & - & - & 16 & 27.1 \\
\hline Vancomycin & 45 & 72.6 & - & - & 17 & 27.4 \\
\hline
\end{tabular}

Table 4. Microorganisms isolated from stool.

\begin{tabular}{|c|c|c|}
\hline Isolates & Number & $\%$ \\
\hline Enterococcus faecium & 14 & 32.6 \\
\hline Enterococcus faecalis & 7 & 16.3 \\
\hline Escherichia coli & 5 & 11.6 \\
\hline Candida & 6 & 14.0 \\
\hline Klebsiella pneumoniae & 3 & 7.0 \\
\hline Enterococcus avium & 3 & 7.0 \\
\hline Enterobacter cloacae & 1 & 2.3 \\
\hline Pseudomonas aeruginosa & 1 & 2.3 \\
\hline Staphylococcus aureus & 1 & 2.3 \\
\hline Staphylococcus haemolyticus & 1 & 2.3 \\
\hline Hafnia alvei & 1 & 2.3 \\
\hline Total & 43 & \\
\hline
\end{tabular}

\section{Stool}

A total of 43 microorganisms were isolated from feces (Table 4). Twenty-six (60.5\%) were gram-positive, 11 (25.5\%) were gramnegative strains, and 6 (14.0\%) were Candida species. The most common bacteria were Enterococcus faecium (32.6\%), Enterococcus faecalis (16.3\%), and Escherichia coli (11.6\%).

\section{Comparison between bile cultures and stool cultures}

In $24.1 \%$ of patients, all microorganisms found in bile could also been found in stool. In $75.9 \%$ of cases, at least 1 microorganism found in bile was also found in stool and in $24.1 \%$ of patients, microorganisms found in bile could not been found in stool.

\section{Discussion}

Diagnosis and therapy of biliary complications after LT is a domain of ERCP. However, ERCP is an invasive procedure and can lead to several severe complications, especially if further interventions (such as sphincterotomy, balloon dilatation, and/ or endoscopic drain placement) are required. Some of these dreaded complications are septic in nature [12-14]. In our study, we analyzed the spectrum of isolates found in bile of liver transplant recipients during ERCP and tested their sensitivity to given antibiotic prophylaxis.

Septic complications such as cholangitis are dreaded after ERCP in liver transplant population. A starting point for the bile colonization is the intestine. From there, ascending microorganisms can lead to septic complications, especially in case of biliary 
obstruction and/or immunosuppression [15-17]. Negm et al. identified liver transplantation as a risk factor for biliary colonization with microorganisms. In the same study, Negm et al. showed the clinical relevance of collected bile cultures during ERCP in case of complicating cholangitis [8]. Furthermore, Barkholt et al. showed in 1997 that intestinal flora represents a reservoir for ascending cholangitis and other infections, especially after liver transplantation [11].

Our results show a completely different spectrum of microorganisms found in bile of liver transplant recipients in comparison with that found in bile of immunocompetent non-transplant individuals. In our study, in $64.2 \%$ of cases we found gram-positive bacteria, in $22.2 \%$ gram-negative bacteria, and in $13.5 \%$ Candida. Most common isolates were Enterococcus species, whereas, in the non-transplant setting, more gram-negative bacteria, especially E. coli and klebsiella, are found [18-21]. Our results confirm former studies that showed a shift in microbial spectrum in bile of immunosuppressed patients after LT towards gram-positive bacteria with a markedly increased incidence of Enterococci [8-10]. Those findings emphasize the relevance of analysis of bile isolates in liver transplant recipients with regard to the spectrum and resistogram to select an effective antibiotic prophylaxis for these patients and also to treat potential septic complications after ERCP in liver transplant patients.

The use of only vancomycin or linezolid would have covered nearly $73 \%$ of the detected bacteria. The addition of vancomycin or linezolid to the originally administered antibiotics would have covered more than $95 \%$ of isolates. This is due to the above-mentioned increased incidence of gram-positive bacteria in the bile of liver transplant patients, especially of Enterococci.

In 2006 Millonig et al. found resistance rates to ciprofloxacin, piperacillin / tazobactam, and imipenem of only 19-32\% of bacteria isolated from bile of liver transplant recipients [9]. These resistance rates are lower than those demonstrated in our study. A possible explanation for that is the global increase of acquired antimicrobial resistance among bacteria [22,23].

\section{References:}

1. Ryu CH, Lee SK: Biliary strictures after liver transplantation. Gut Liver, 2011; 5: $133-42$

2. Sanna C, Saracco GM, Reggio D et al: Endoscopic retrograde cholangiopancreatography in patients with biliary complications after orthotopic liver transplantation: outcomes and complications. Transplant Proc, 2009; 41: 1319-21

3. Freeman ML, Nelson DB, Sherman S et al: Complications of endoscopic biliary sphincterotomy. N Engl J Med, 1996; 335: 909-18

4. Loperfido S, Angelini G, Benedetti G et al: Major early complications from diagnostic and therapeutic ERCP: a prospective multicenter study. Gastrointest Endosc, 1998; 48: 1-10

5. Andriulli A, Loperfido S, Napolitano G et al: Incidence rates of post-ERCP complications: a systematic survey of prospective studies. Am J Gastroenterol, 2007; 102: 1781-88
In our study we found a high correlation between microorganisms found in bile and those isolated from stool. This finding, and identifying sphincterotomy as a risk factor for bile colonization, confirm the results of former studies that identify the intestinum as a starting point for ascending colonization of bile and thus for septic complications after ERCP such as cholangitis and sepsis [15-17]. The spectrum of the intestinal flora is based on a continuous interplay between the immune system and microorganisms [24]. Through the influence of immunosuppression after LT, this balance between immune system and gut flora may be disrupted, which can lead to an increased tolerance of gram-positive bacteria, especially enterococci, by the impaired immune system of the gut.

Interestingly, in all patients who developed signs of septicemia with the evidence of positive blood cultures, there was a match between isolates from blood and those from bile.

\section{Conclusions}

In conclusion, bile of liver transplant recipients is predominantly colonized with microorganisms. This colonization is most likely a consequence of ascension of the microorganisms from the gut. Therefore, the monitoring of bile during endoscopic intervention in the biliary tract of liver transplant patients could help to select effective prophylactic antibiotic regimes as well as to facilitate the choice of suitable antimicrobial therapy in case of cholangitis and sepsis. Grampositive bacteria, especially Enterococci, seem to be frequent in bile of liver transplant recipients. Therefore, in case of septic complications after ERCP, empiric antibiotic therapy should include vancomycin or linezolid. Further prospective, large-scale, randomized trials are warranted to confirm our findings and to modulate the generally recommended antibiotic prophylaxis in case of needed endoscopic biliary interventions after LT.

6. Csendes A, Fernandez M, Uribe P: Bacteriology of the gallbladder bile in normal subjects. Am J Surg, 1975; 129: 629-31

7. Csendes A, Becerra M, Burdiles P et al: Bacteriological studies of bile from the gallbladder in patients with carcinoma of the gallbladder, cholelithiasis, common bile duct stones and no gallstones disease. Eur J Surg, 1994; 160: 363-67

8. Negm AA, Schott A, Vonberg RP et al: Routine bile collection for microbiological analysis during cholangiography and its impact on the management of cholangitis. Gastrointest Endosc, 2010; 72: 284-91

9. Millonig G, Buratti T, Graziadei IW et al: Bactobilia after liver transplantation: frequency and antibiotic susceptibility. Liver Transpl, 2008; 12: 747-53

10. Gotthardt DN, Weiss KH, Rupp C, et al. Bacteriobilia and fungibilia are associated with outcome in patients with endoscopic treatment of biliary complications after liver transplantation. Endoscopy, 2013; 45: 890-96 
11. Barkholt LM, Andersson J, Ericzon BG et al: Stool cultures obtained before liver transplantation are useful for choice of perioperative antibiotic prophylaxis. Transpl Int, 1997; 10: 432-38

12. Deviere J, Motte S, Dumonceau JM et al: Septicemia after endoscopic retrograde cholangiopancreatography. Endoscopy, 1990; 22: 72-75

13. Kwak MS, Jang ES, Ryu JK et al: Risk factors of post endoscopic retrograde cholangiopancreatography bacteremia. Gut Liver, 2013; 7: 228-33

14. Anderson DJ, Shimpi RA, MCDonald JR et al: Infectious complications following endoscopic retrograde cholangiopancreatography: an automated surveillance system for detecting postprocedure bacteremia. Am J Infect Control, 20018; 36: 592-94

15. Hanau LH, Steigbigel NH: Acute (ascending) cholangitis. Infect Dis Clin North Am, 2000; 14: 521-46

16. Lipsett PA, Pitt HA: Acute cholangitis. Front Biosci, 2003; 8: s1229-39

17. Muir CA: Acute ascending cholangitis. Clin J Oncol Nurs, 2004; 8: 157-60

18. Chang WT, Lee KT, Wang SR et al: Bacteriology and antimicrobial susceptibility in biliary tract disease: an audit of 10-year's experience. Kaohsiung J Med Sci, 2002; 18: 221-28
19. Flores C, Maguilnik I, Hadlich E, Goldani LZ: Microbiology of choledochal bile in patients with choledocholithiasis admitted to a tertiary hospital. Gastroenterol Hepatol, 2003; 18: 333-36

20. Kawai K, Akasaka Y, Murakami K et al: Endoscopic sphincterotomy of the ampulla of Vater. Gastrointest Endosc, 1974; 20: 148-51

21. Salvador VB, Lozada MC, Consunji RJ: Microbiology and antibiotic susceptibility of organisms in bile cultures from patients with and without cholangitis at an Asian academic medical center. Surg Infect (Larchmt), 2011; 12: 105-11

22. Gould IM: Antibiotic resistance: the perfect storm. Int J Antimicrob Agents, 2009; 34 Suppl 3: S2-5

23. Noll I, Schweickert B, Abu Sin M et al. [Antimicrobial resistance in Germany. Four years of antimicrobial resistance surveillance (ARS)] Bundesgesundheitsblatt Gesundheitsforschung Gesundheitsschutz, 2012; 55: 1370-76

24. Marchesi J, Shanahan F: The normal intestinal microbiota. Curr Opin Infect Dis, 2007; 20(5): 508-13 